\section{Patología inflamatoria ocular: coriorretinitis serpinginosa}

\section{Ocular inflammatory diseases: serpinginous choroidopathy}

\section{Resumen}

Dentro de la patología inflamatoria ocular, la coriorretinitis serpinginosa es un tipo de uveitis posterior que afecta a la coroides y al epitelio pigmentario de la retina. Es una entidad de muy baja prevalencia pero con un curso recurrente y progresivo que puede llevar a la pérdida irreversible de la agudeza visual. Presentamos dos casos con afectación inflamatoria característica así como el tratamiento y pronóstico con el que cursaron y se comenta la literatura más reciente respecto a las características demográficas y clínicas de las diferentes series de casos publicados así como una actualización terapéutica.

\section{Abstract}

Serpiginous choroiditis is a type of posterior uveitis affecting choroid and retinal pigment epithelium. It is a very low prevalence disease but with a recurrent and progressive course leading to irreversible loss of visual acuity. We present two cases with characteristic inflammatory involvement as well as treatment and prognosis. We reviewed the demographic and clinical characteristics of published case series and an update on treatment.

Keywords: coriorretinitis serpinginosa; uveitis; patologia inflamatoria ocular; serpinginous choroidopathy; uveitis; ocular inflammatory diseases.

\section{Pablo Antonio Zurita Prada*}

Claudia Lia Urrego Laurin* Isabel Izquierdo Millán**

\footnotetext{
* Area de Reumatología

Complejo Hospitalario de Segovia

** Servicio de Oftalmología

Complejo Hospitalario de Segovia

Correspondencia:
}

\section{Pablo Antonio Zurita Prada}

झ pzprada@yahoo.es 


\section{Introducción}

La Coriorretinitis Serpinginosa (CS) es una entidad inflamatoria ocular de muy baja incidencia enmarcada en el contexto de las uveitis posteriores donde el proceso inflamatorio se localiza inicialmente a nivel de la capa coroidea. Se desconoce su etiología, implicándose teorías etiopatogénicas autoinmunes e infecciosas. Sin tratamiento suele comportar un pronóstico grave pues el proceso inflamatorio tiende a presentar un curso progresivo, crónico y recurrente con deterioro o pérdida de la agudeza visual.

\section{Caso 1}

Mujer de 71 años sin antecedentes de interés salvo dislipemia y diabetes mellitus no insulindependiente que en Febrero de 2010 presentó pérdida de visión en su ojo derecho. En la exploración oftalmológica se apreciaba una agudeza visual (AV) en su ojo derecho (OD) de "cuenta dedos a un metro" y en el izquierdo (OI) de 1, siendo la presión intraocular de 13 y $15 \mathrm{~mm}$ de $\mathrm{Hg}$ respectivamente. En la biomicroscopía se observaban sendas cataratas nucleares que no justificaban la agudeza visual del OD, sin apreciarse fenómeno Tyndall. El fondo de ojo presentaba en ambos ojos lesiones atróficas peripa- pilares de forma geográfica, que en el OD llegaban a afectar al área macular, con membrana neovascularsubretiniana en esa zona. En la tomografía óptica de coherencia (OCT) macular del OD aparecía una imagen de edema macular quístico (EMQ) y en la angiofluoresceíngrafía (AFG) del OD se observaba hipofluorescencia precoz debido al bloqueo de fluoresceína por el epitelio pigmentario retiniano edematoso e hiperfluorescencia tardía a partir de los bordes de la lesión activa (Figura 1A y 1B). De las pruebas complementarias realizadas presentaba un hemograma y bioquímica normales salvo glucosa 132; estudio inmunológico (FR, CCP, ANA, DNA, ANCA, Crioglobulinas) negativo, estudio serológico (toxoplasma, LUES, CMV) negativo; tipaje HLA B27 y B51 negativo; Mantoux negativo y Rx de tórax y pelvis con caderas sin alteraciones significativas. Ante la sospecha clínica de coroiditis serpiginosa se inició tratamiento con dosis altas esteroideas (prednisona $1 \mathrm{mg} / \mathrm{Kg} / \mathrm{día}$ ) junto con ciclosporina (5 mg/ $\mathrm{Kg} / \mathrm{día}$ ). A las seis semanas de iniciar el tratamiento, debido a intolerancia comprobada e HTA, se sustituyó la Ciclosporina por Azatioprina $(1,5 \mathrm{mg} / \mathrm{Kg} /$ día según niveles TMPT) y se añadió profilaxis con alendronato y suplementos de Calcio y Vitamina D. Con dicho tratamiento y a pesar de no recuperar

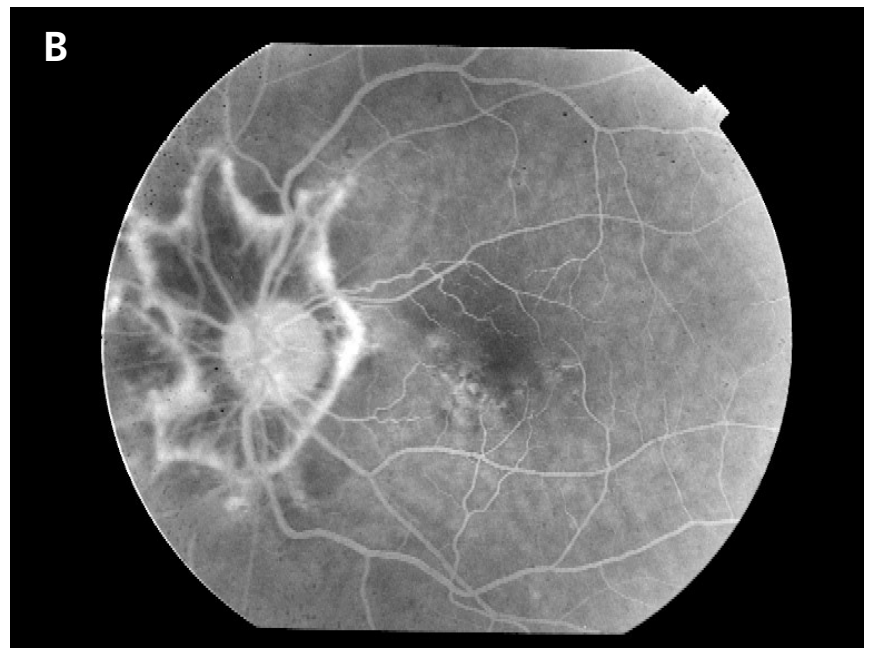

Figura 1A. Angiofluoresceingrafía de ojo derecho. 1B. Angiofluoresceingrafía de ojo izquierdo. 
agudeza visual, se observó mejoría de las lesiones oftalmológicas peripapilares y disminución del EMQ. A los 3 meses de tratamiento, se administró una inyección intravítrea de ranibizumab (Lucentis®) en OD para intentar mejorar la neovascularización macular, obteniendo una leve recuperación de la AV a 0,1 . Se inició un descenso progresivo del tratamiento esteroideo hasta su suspensión a los 10 meses de su inicio. A los 12 meses de tratamiento, en el contexto de monoterapia con azatioprina, presentó empeoramiento del EMQ sin otros signos de actividad, por lo que se realizó una inyección intravítrea de acetónido de triamcinolona (Trigón depot) en OD, con mejoría anatómica pero sin recuperación funcional. Se mantuvo la azatioprina completando dos años de tratamiento inmunosupresor. Con dicha estrategia y hasta junio del 2015, la paciente ha mantenido lesiones serpiginosas estables sin signos de actividad, pero con poca recuperación de la agudeza visual en su OD por la afectación macular, con EMQ y fibrosis macular como secuelas. (Figura 2A y 2B)

\section{Caso 2}

Varón de 62 años sin antecedentes personales de interés, que en Agosto del 2005 acudió a la consulta de Oftalmología por disminución de la visión en su OI. En la exploración se observaba una AV de 1 en OD y de "cuenta dedos a un metro" en OI. En el fondo de ojo se apreciaba una imagen compatible con coroiditis serpiginosa en ambos ojos, con EMQ en OI. La AFG del OD no se apreciaba signos de actividad y la del OI mostraba hipofluorescencia precoz e hiperfluorescencia tardía a partir de los bordes de la lesión (Figura 3). El estudio inmunológico y la serología infecciosa resultaron negativas salvo serología de Toxoplasma IgG positivo e IgM negativo. En la radiografía de tórax aparecían lesiones cicatriciales residuales en lóbulo superior derecho y el Mantoux era positivo. Se instauró tratamiento con Prednisona $(0.5 \mathrm{mg} / \mathrm{kg} / \mathrm{d}$ ía) e Isoniacida $300 \mathrm{mg} / \mathrm{día}$ que se mantuvo durante nueve meses. A las 3 semanas de iniciado el tratamiento, se observaba en el fondo del ojo edema retiniano en los bordes de la lesión en OI y EMQ, por lo que se inyectó acetónido de triamcinolona (Trigón depot peribulbar) en OI, mejorando el edema retiniano pero con persistencia del EMQ. En diciembre 2005, se consultó con el Servicio de Reumatología añadiéndose Ciclosporina $(5 \mathrm{mg} / \mathrm{Kg} /$ día) remitiendo al mes los signos de actividad de las lesiónes serpinginosas. Seis meses después, la agudeza visual del OD era de 1 y la del Ol había mejorado a 0,05. En una nueva AFG de junio 2006,
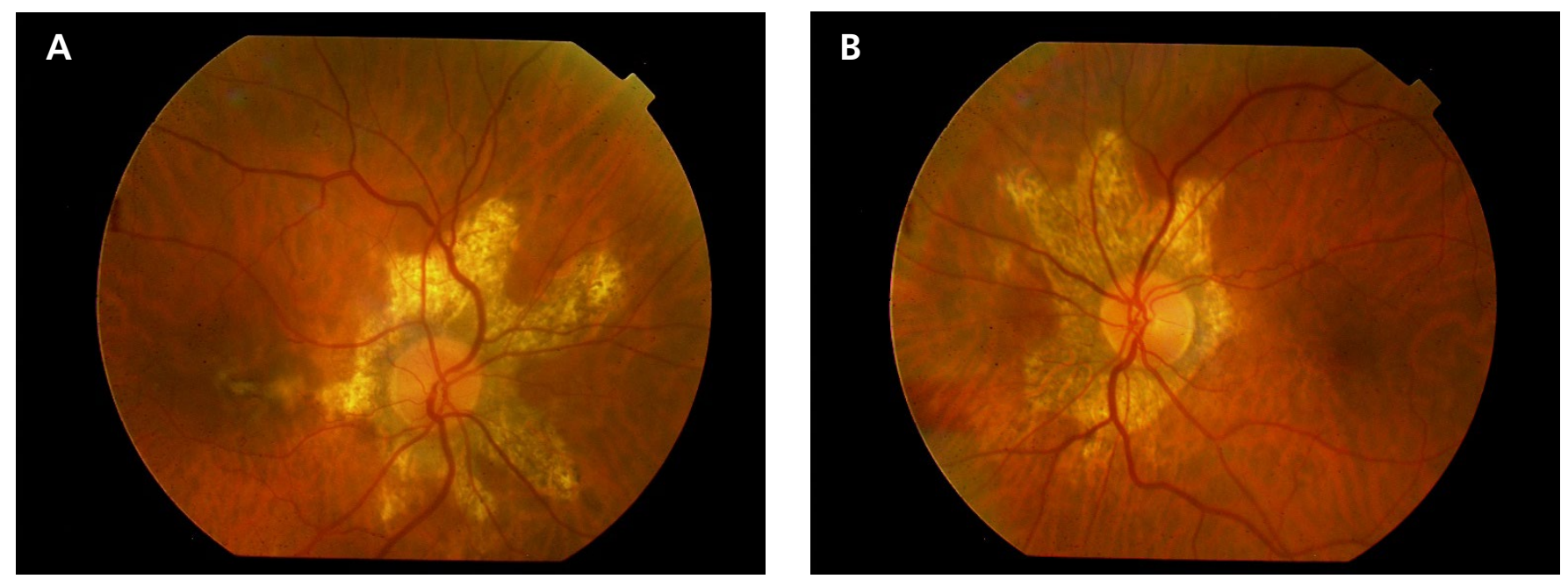

Figura 2A. Retinografía de ojo derecho. 2B. Retinografía de ojo izquierdo. 


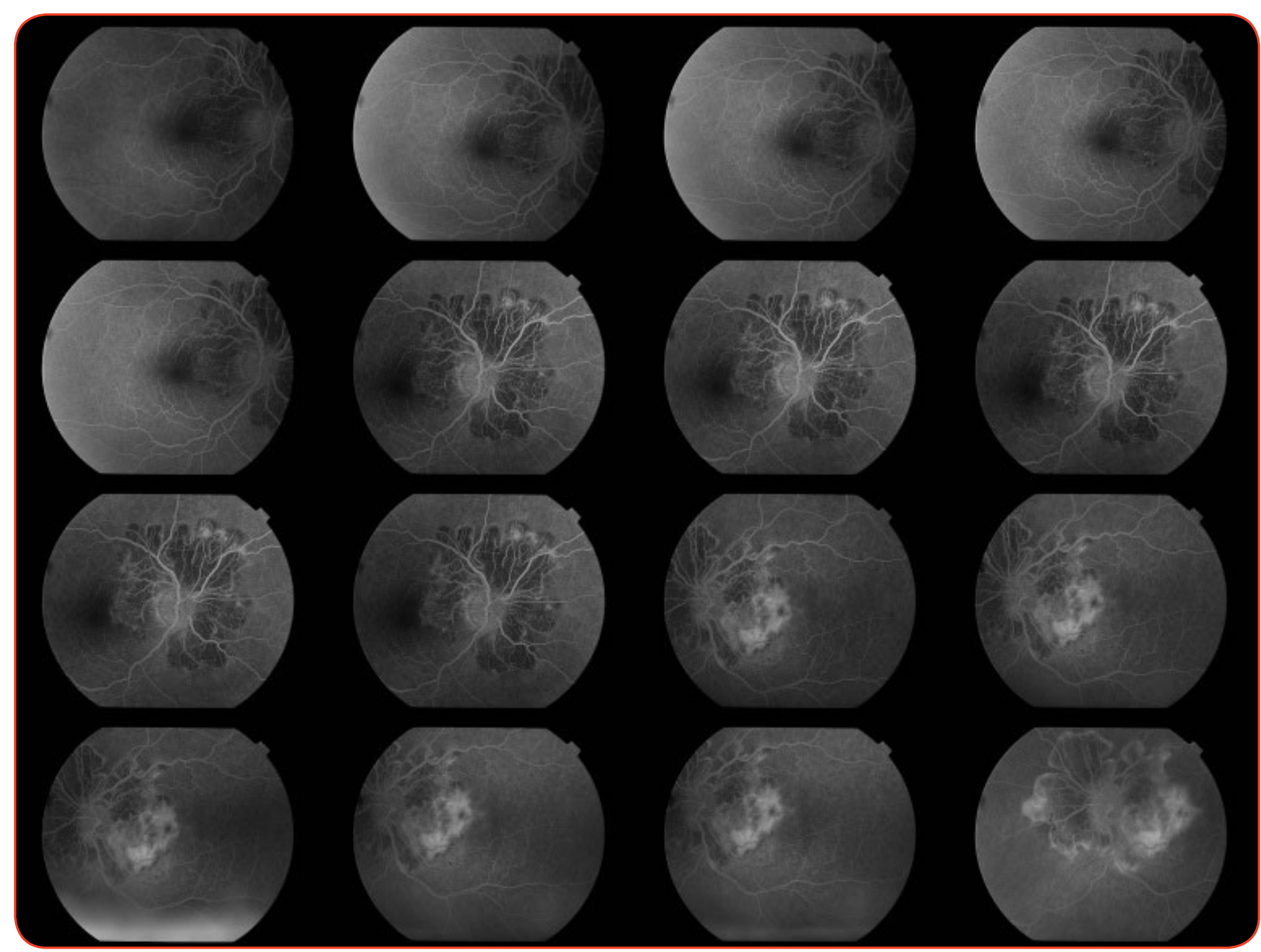

Figura 3. Angiofluoresceingrafía de ambos ojos

no se objetivaron signos de actividad, presentando hiperfluorescencia en el límite entre las zonas sanas y las lesiones. En el fondo de ojo se apreciaban las lesiones atróficas peripapilares con afectación del área macular en OI y presencia de fibrosis. Se disminuyó progresivamente el tratamiento inmunosupresor, suspendiéndose ciclosporina en diciembre 2006 y bajas dosis esteroideas de mantenimiento en marzo 2007. Hasta la actualidad, las lesiones se han mantenido inactivas (Figura 4).

\section{Discusión}

La Coriorretinitis Serpinginosa (CS) es un tipo de uveitis posterior caracterizada por una inflamación crónica, recurrente y recidivante de la membrana coroidea, los capilares coroideos y el epitelio pigmentario de la retina. Ha sido documentada en la literatura de muy diferentes maneras y distintas nomenclaturas: coroiditis geográfica, coroidopatía geográfica, coroidopatía peripapilar coroidal, siendo Laatikainen ${ }^{1}$ el que probablemente completó mejor la descripción que actualmente se acepta y que permanece clasificada separadamente y diferenciada de los síndromes de puntos blancos ("white dot syndromes" $)^{2}$. Es una entidad de muy baja prevalencia, calculándose que puede corresponder a menos del $5 \%$ del total de las uveitis posteriores. Se ha descrito, sin embargo, una mayor incidencia en ciertas regiones geográficas de la India ${ }^{3}$. Su etiología 


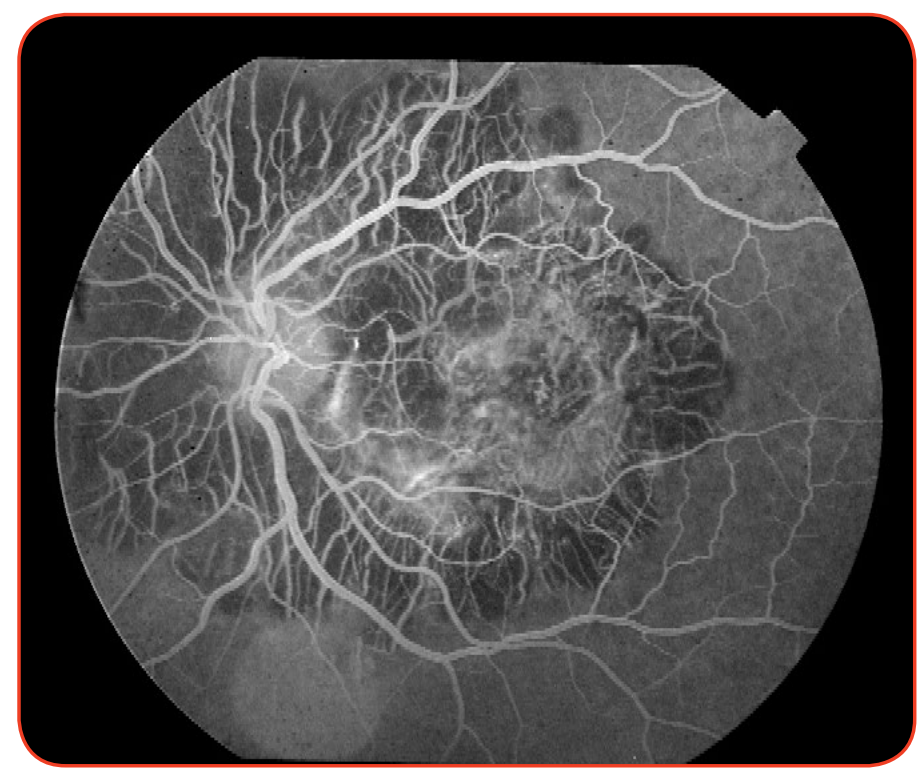

Figura 4. Angiofluoresceingrafía de ojo izquierdo.

permanece desconocida aunque se han implicado factores etiopatogénicos de causas autoinmune e infecciosas, destacando entre estas últimas la etiología tuberculosa. Se han descrito casos aislados asociados a enfermedades como la sarcoidosis ${ }^{4}$ enfermedad de Crohn $^{5}$, panarteritis nodosa ${ }^{6}$, lupus eritematoso sistémico ${ }^{7}$, cáncer de cervix ${ }^{8}$ o hepatitis autoinmune ${ }^{9}$.

En la descripción clásica², las lesiones se inician a nivel papilar y se distribuyen de manera centrífuga, en forma de parches mal definidos de color amarillo grisáceo o cremoso. Estas imágenes se corresponden con infiltrados subretinianos originarios en la región peripapilar y progresan de forma serpentina irregular centrífuga que originan el aspecto típico de la enfermedad. La retina suprayacente es por lo general edematosa y ocasionalmente puede ocurrir un desprendimiento seroso. Cuando las lesiones activas se resuelven, con o sin tratamiento, dejan un área de atrofia tanto del tejido coriocapilar, el epitelio pigmentario y la retina suprayacente. En un mismo ojo, pueden observarse lesiones múltiples en diferentes etapas de resolución. La enfermedad se caracteriza por múltiples recurrencias a intervalos variables, que se producen, por lo general, en los bordes anteriores de las cicatrices atróficas. Con cada nuevo episodio de actividad, hay una extensión del daño coriocapilar y atrofia del epitelio pigmentario de la retina en forma de un patrón geográfico. Además de esta forma de presentación clásica, pueden existir afectaciones anatómicas aisladas de la mácula o únicamente periféricas ${ }^{10}$. Las complicaciones más temidas son la afectación macular, el edema macular quístico y la aparición de neovascularización subretiniana (NVSR) que se relaciona principalmentecon una mayor tasa de recurrencias. Los casos crónicos finalizan con atrofia coriorretiniana y fibrosis subretiniana.

Debido a su baja incidencia con excepción de algunas zonas geográficas, existe heterogeneidad en las características sociodemográficas y clínicas que exponemos en la Tabla 1, basadas en la revisión de los datos de las publicaciones de series de pacientes $^{11,12,13,14,15}$. Suele existir preponderancia en el sexo masculino, la edad media de la vida y la afectación bilateral. Cerca del $50 \%$ de los casos van a presentar afectación macular al inicio o durante el curso de la enfermedad. La mayoría de los casos han sido tratados con corticoterapia o en combinación con inmunosupresores y existen casos puntuales aislados tratados con antituberculosos.

La CS suele ser un síndrome inflamatorio ocular donde la asociación con enfermedad sistémica autoinmune es incidental, por lo que el papel del Reumatólogo, después de haber realizado el despistaje protocolario habitual, consistirá en la mayoría de los casos en el apoyo para el control y la optimización del tratamiento corticoideo e inmunosupresor. Sin embargo, debe advertirse que para la CS es necesaria una mayor atención en el despistaje de la enfermedad tuberculosa. Esto se debe a que la mayor incidencia de CS en áreas endémicas de tuberculosis ha llevado a algunos autores a exponer la hipótesis de la etiología tuberculosa, idea reforzada además por el éxito y la curación de algunos casos puntuales con tratamiento antituberculoso. A pesar de ello, esta sospecha no ha sido confirmada y Saurabhet no encuentra diferencias ni en la forma 
Tabla 1.

\begin{tabular}{|c|c|c|c|c|c|}
\hline & $\begin{array}{c}\text { Marcuello et al. }{ }^{11} \\
\text { (2004) }\end{array}$ & $\begin{array}{c}\text { Abrez et al. }{ }^{12} \\
(2007)\end{array}$ & $\begin{array}{c}\text { Saurabhet et al. }{ }^{13} \\
\text { (2013) }\end{array}$ & $\begin{array}{c}\text { Toniolo et al. }{ }^{14} \\
\qquad(2014)\end{array}$ & $\begin{array}{c}\text { Balarabe et al. }{ }^{15} \\
\text { (2014) }\end{array}$ \\
\hline $\mathrm{N}^{\circ}$ de pacientes & 11 & 70 & 54 & 18 & 17 \\
\hline $\mathrm{N}^{\circ}$ de ojos & 20 & 107 & 91 & 27 & 29 \\
\hline Raza & España & India & India & N. Zelanda y Australia & India \\
\hline Sexo & $90 \%$ varones & $70 \%$ varones & $64.8 \%$ varones & $50 \%$ varones & $82.4 \%$ varones \\
\hline Edad media & 56 años & 30.3 años & 34.1 años & 47.7 años & 31 años \\
\hline Bilateralidad & $81.1 \%$ & $52.9 \%$ & $68.5 \%$ & $61 \%$ & $64.7 \%$ \\
\hline Vitritis & $9 \%$ & $1.4 \%$ & $0 \%$ & $33 \%$ & $0 \%$ \\
\hline Afectación macular & $35 \%$ & $84 \%$ & $41.75 \%$ & $55 \%$ & $24.1 \%$ \\
\hline NVSR & $45 \%$ & $0.9 \%$ & $2.56 \%$ & $33 \%$ & $\mathrm{NE}_{1}$ \\
\hline QFN y/o mantoux + & 0 casos & 5 casos & 12 casos & 5 casos & 1 caso \\
\hline Recaidas & $91 \%$ & $45.7 \%$ & $18 \%$ & $77 \%$ & $65 \%$ \\
\hline Tto. corticoideo & $100 \%$ & $100 \%$ & $94.4 \%$ & $100 \%$ & $100 \%$ \\
\hline Tto. inmunosupresor & $27.27 \%$ & $\mathrm{NE}_{2}$ & $29.62 \%$ & $\mathrm{NE}_{2}$ & $64.7 \%$ \\
\hline Tto. antituberculoso. & 0 & 5 & 0 & 4 & 1 \\
\hline
\end{tabular}

de presentación, gravedad ni en el pronóstico con tratamiento inmunosupresor cuando se diferencia a los pacientes por despistaje tuberculoso (pacientes con mantoux negativo versus mantoux positivo) ${ }^{13}$. Respecto al éxito de los pacientes tratados con tratamiento antituberculoso, debe tenerse en cuenta que el diagnóstico de la CS sigue siendo clínico y descriptivo, donde los hallazgos diferenciales entre la CS y una verdadera coriorretinitis multifocal tuberculosa pueden suponer un reto y dilema diagnóstico para el Oftalmólogo. En base a la experiencia reportada, en aquellos casos con duda diagnóstica y sin datos de TBC activa, pueden tenerse en cuenta unos criterios diferenciales que pueden orientar a la etiología tuberculosa: paciente perteneciente a un área endémica o haber tenido un contacto reciente de riesgo tuberculoso, mantoux positivo, presencia de células en la cámara anterior y vítreo, lesiones multifocales con independencia de afectación del polo posterior y ausencia de respuesta a GC e inmunosupresores, 16,17,18.
El pronóstico de la CS viene determinado principalmente por la afectación de la agudeza visual así como la aparición de complicaciones. En la serie de Saurabhet ${ }^{13}$, el $72 \%$ de los casos presentan una AV igual o mejor de 20/60 al final de seguimiento. En la serie de 11 pacientes de Marcuello, hasta un $45 \%$ de los ojos afectos manifestaron una agudeza visual final menor de 0,1 a pesar del tratamiento ${ }^{11}$.Otros autores, basándose en la existencia de formas variantes sin afectación macular pero con importantes escotomas o defectos campimétricos en la exploración ocular, advierten de la necesidad de la selección de otros parámetros distintos de la AV para vigilar correctamente la actividad o la reaparición de recidivas durante el curso evolutivo ${ }^{13,14}$. A este respecto, Marcuello destacaba que, en el inicio de la fase aguda, 6 de los 11 pacientes presentaban placas antiguas de atrofia coriorretiniana y dispersión pigmentaria en el polo posterior en el ojo contralateral correspondientes a episodios antiguos asintomáticos de la enfermedad. Nuestros dos 
pacientes no presentaban lesiones periféricas pero sí en el ojo contralateral asintomático de cronología no determinada sin deterioro de la AV que habrían pasado desapercibidas.

La estrategia del tratamiento de la CS se basa en controlar los síntomas inflamatorios agudos, prevenir las recurrencias y afrontar las complicaciones secundarias, siendo las más graves entre estas últimas la afectación macular, el EMQ y la NVSR, pues son la causa principal de pérdida irreversible de la agudeza visual, situación que lamentablemente también han padecido nuestros dos casos.

El tratamiento más habitualmente utilizado de la CS es la terapia corticoidea sistémica. Ha demostrado controlar los brotes inflamatorios y prevenir las recurrencias. Abrez et al ${ }^{12}$. utiliza la administración de pulsos de metilprednisolona (MP iv) de manera protocolaria seguida de disminución progresiva de prednisona oral posteriormente. En otras series, el uso de MP iv es más selectivo, predominando corticoterapia oral a alta dosis. Los tratamientos inmunosupresores se han indicado inicialmente para intentar disminuir el número de recurrencias ${ }^{19}$, pero su uso en la actualidad no se encuentra completamente estandarizado, siendo muchos los autores que sólo los reservan para las presentaciones más graves (con pérdida de agudeza visual y afectación macular), refractarias, recurrentes o donde predominan claramente los efectos secundarios esteroideos. La azatriopina (AZA), ciclosporina (CyP), ambas en combinación (triple terapia: esteroides + AZA + CyP) así como el micofenolato mofetilo han sido los más utilizados ${ }^{20,21,22}$. Con menor frecuencia se encuentra el uso de agentes alquilantes como el clorambucilo o la ciclofosfami$\mathrm{da}^{23}$. No existen ensayos clínicos aleatorizados, por lo que ni la dosis ni la duración ha sido estandarizada, siendo siempre a criterio del clínico en la experiencia publicada. Se han comunicado más recientemente experiencias de casos refractarios con adalimumab e infliximab24,25. Debe advertirse al respecto que en un paciente con infliximab, el paciente falleció posteriormente en el seno de una tuberculosis diseminada ${ }^{26}$, por lo que parece prudente en el caso de la CS (y su relación con la tuberculosis) extremar la precaución con el uso de terapia anti-TNF como una alternativa a la que habitualmente estamos dispuestos a recurrir en otros casos de uveitis refractarias. Las inyecciones locales corticoideas (intravitreas, peribulbares, subtenianas) son recomendadas en casos graves y como coadyuvantes al tratamiento sistémico. Respecto a la NVSR como complicación específica de CS, el tratamiento con laser e inhibidores del factor de crecimiento endotelial vascular (ranibizumab y bevacizumab) han sido documentados recientemente con resultados favorables 27,28 .

\section{Conclusión}

En resumen, la CS es una uveitis posterior de curso grave y recurrente de predominio en edad media de la vida, de muy baja prevalencia excepto en algunas áreas endémicas de tuberculosis, siendo necesario una mayor atención al diagnóstico diferencial con esta afectación ocular infecciosa. Su detección requiere el rápido abordaje para evitar la pérdida de agudeza visual y la aparición de secuelas. El tratamiento se basa principalmente en el uso de corticoterapia e inmunosupresores, siendo sólo algunos casos seleccionados los que se podrían beneficiar de tratamiento antituberculoso.

\section{Bibliografía}

1. Laatikainen L, Erkkila H. Serpiginous choroiditis. Br J Ophthalmol. 1974; 58: 777-83.

2. R. Nussenblatt and S. Whitcup. Uveitis. Fundamentals and clinical practice. $4^{\circ}$ Edition.

3. Gupta V, Agarwal A, Gupta A, Bambery P, Narang S. Clinical characteristics of serpiginous choroidopathy in North India. Am J Ophthalmol2002; 134: 47-56.

4. Edelsten C, Stanford MR, Graham EM: Serpiginous choroiditis: an unusual presentation of ocular sarcoidosis. Br J Ophthalmol 78:70-1, 1994

5. Ugarte M, Wearne IM: Serpiginous choroidopathy: an unusual association with Crohn's disease. Clin Experiment Ophthalmol 30:437-9, 2002 
6. Pinto Ferreira F, Faria A, Ganhão F. Periarteritis nodosa with initial ocular involvement. J Fr Ophtalmol1995; 18:788-93,

7. Fuentes- Paez G, Cerlis Sanchez J, Torres J, Martinez Osorio H, Herreras JM. Sepiginous choroiditis in a patient with systemic lupus erythematosus. Lupus 2005; 14:928-9

8. Jordano Perez JJ, Córdoba Lorenzo M, Ruiz Lomas C, Márquez Baez FJ, Ortega Ortiz A. Serpinginus choroiditis in a patient with uterine cervix carcinoma. Arch Soc Esp Oftalmol 2012; 87: 86-9

9. Gómez Maestra MJ, Francés E, Ausín E, Martinez-Costa R. autoimmune hepatitis in a patient with serpiginous choroiditis. Arch Soc Esp Oftalmol 2007; 82:773-6

10. Lim WK, Buggage RR, Nussenblatt RB. Serpiginous choroiditis. Surv Ophthalmol 2005; 50: 231-44.

11. Marcuello Melendo B, Torrón Fernández-Blanco C, Pérez Oliván S, Ruiz Moreno O, Ferrer Novella E, Honrubia López FM. Serpiginous choroiditis: clinical course and treatment. Arch Soc Esp Oftalmol. 2004 May; 79(5):237-42

12. Abrez H, Biswas J, Sudharshan S. Clinical profile, treatment, and visual outcome of serpiginous choroiditis. Ocullmmunol Inflamm 2007; 15: 325-35.

13. Saurabh K, Panigrahi PK, Kumar A, Roy R, Biswas. Profile of serpiginous choroiditis in a tertiary eye care centre in eastern India. Indian J Ophthalmol. 2013 Nov; 61(11):649-52.

14. Toniolo J, Hall AJ, Sims J, Fraser-Bell S, Khan J, Younan C, KentSmith B, Young S, Paul E, Lim LL. Retrospective analysis of the natural history and management of serpiginous choroiditis in Australia and New Zealand. Clin Experiment Ophthalmol. 2014 Sep-Oct; 42(7):656-64

15. Balarabe $A H$, Biswas J. Serpiginous choroiditis in a referral clinic in India: visual field changes and clinical correlates. Ocul Immuno IInflamm. 2014 Oct; 22(5):379-83.

16. Bansal R, Gupta A, Gupta V, Dogra MR, Sharma A,Bambery P. Tubercular serpiginous-like coroiditis presenting as multifocal Serpiginoid choroiditis. Ophthalmology2012; 119: 2334-42.

17. Khanamiri HN, Rao NA. Serpiginous choroiditis and infectious multifocal serpiginoid choroiditis. Surv Ophthalmol 2013; 58: 203-32.

18. Vasconcelos-Santos DV, Rao PK, Davies JB, Sohn EH, Rao NA. Clinical features of tuberculous serpiginous like choroiditis in contrast to classic Serpiginous choroiditis. Arch Ophthalmol 2010; 128: 853-8.

19. Christmas NJ, Oh KT, Oh DM, Folk JC. Long-term follow-up of patients with serpinginous choroiditis. Retina 2002; 22: 550-6.

20. Vianna RN, Ozdal PC, Deschenes J, Burnier MN, Jr. Combination of azathioprine and corticosteroids in the treatment of Serpiginous choroiditis. Can J Ophthalmol2006; 41: 183-9.

21. Araujo AA, Wells AP, Dick AD, Forrester JV. Early treatment with cyclosporin in Serpiginous choroidopathy maintains remission and good visual outcome. Br J Ophthalmol 2000; 84: 979-82.

22. Hooper PL, Kaplan HJ. Triple agent immunosuppression in Serpiginous choroiditis. Ophthalmology 1991; 98: 944-51. discussion 51-2.

23. Akpek EK, Jabs DA, Tessler HH, et al.: Successful treatment of Serpiginous choroiditis with alkylating agents. Ophthalmology 2002, 109: 1506-1513
24. Chinchurreta Capote $A$, Requena Jiménez JM, Lorenzo Soto M, Romero Gómez C, García de Lucas MD. Effectiveness of adalimumab for refractory serpiginous choroiditis. Ocul Immunol Inflamm. 2014 Oct;22(5):405-8.

25. Seve P, Mennesson E, Grange JD, Broussolle C, Kodjikian L. Infliximab in Serpiginous choroiditis. Acta Ophthalmol. 2010 Dec; 88(8):e 342-3.

26. Cordero-Coma M, Benito MF, Hernandez AM, Antolin SC \& Ruiz JM (2008): Serpiginous choroiditis. Ophthalmology. 2008 Sep;115(9):1633, 1633.e1-2.

27. D'Ambrosio E, Tortorella P, lannetti L. Management of uveitisrelated choroidal neovascularization: from the pathogenesis to the therapy. J Ophthalmol. 2014; 2014:450428.

28. Parodi MB, lacono $P$, La Spina $C$, Knutsson KA, Mansour A, Arevalo JF, Bandello F. Intravitreal bevacizumab for choroidal neovascularisation in serpiginous choroiditis $\mathrm{Br} J$ Ophthalmol 2014;98:519-522.

\section{Opina sobre este artículo:}
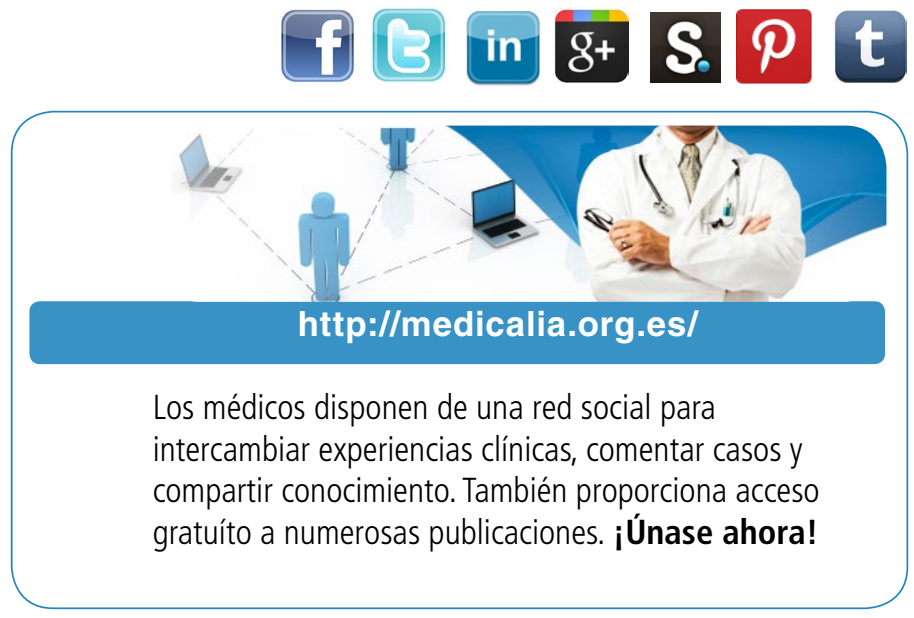

\section{Publish with iMedPub}

\section{http://www.imed.pub}

Acta Reumatológica es una revista que tiene por fin la difusión de estudios clínicos relacionados con aspectos prácticos del diagnóstico, tratamiento y seguimiento de pacientes con patología reumatológica, de estudios epidemiológicos relacionados con patología inflamatoria y musculoesquelética de presentación común o infrecuente en la práctica clínica tanto en población adulta como pediátrica, de casos clínicos de patología poco habitual o de presentaciones inhabituales de patología frecuente, de imágenes didácticas e ilustrativas en reumatología y del estado actual e innovación en la formación especializada en reumatología. 